\title{
Is Antiplatelet Therapy a Risk Factor for Extraction of Tooth?
}

\section{Budhani $S^{1}$, Manekar VS ${ }^{2 *}$ and Datarkar $\mathrm{AN}^{3}$}

${ }^{1} \mathrm{BDS}$, Government Dental College \& Hospital, India

${ }^{2}$ Associate Professor, Department of Oral \& Maxillofacial Surgery, Government Dental College

\& Hospital, India

${ }^{3}$ Professor and Head Department of Oral \& Maxillofacial Surgery, Government Dental College

\& Hospital, India

*Corresponding author: Varsha S Manekar, Associate Professor, Department of Oral \& Maxillofacial Surgery, Government Dental College \& Hospital, Nagpur 440022, India, Tel: 9823077626; Email: varsha_manekar@yahoo.co.in

\section{Abstract}

Medical physicians often advise patients on oral antiplatelet therapy to stop or alter their medication before extraction. A review of the available literature shows that there is an increased risk of cardiovascular episodes if medication is altered or discontinued. Hence there is a need to evaluate the risk of bleeding in patients whom antiplatelet therapy is not discontinued before extraction.

Aim: To evaluate the risk of bleeding in patients in whom oral antiplatelet therapy is not withdrawn prior to extraction of teeth.

Objectives: To evaluate the incidence of intra-operative bleeding. To evaluate the incidence of post-operative bleeding. To evaluate the bleeding profile.

Materials and Methods: This is clinical trial of sample size of 100 participants, were equally divided into two groups :Group A -patients in whom oral anti -platelet therapy was stopped prior to extraction and Group B -patients in whom anti-platelet therapy was continued prior to and following extraction. Physician's fitness consent and informed consent from patients were obtained. Intra-alveolar extractions were performed. The risk of bleeding was observed by incidence of bleeding observed at three stages and patients were monitored. The bleeding was graded as mild, moderate $\&$ severe according to self-introduced scale.

Results: Majority of the patients suffered from mild bleeding (stage I and stage II) which was easily controlled by normal pressure pack. Only $4 \%$ patients (stage I) and $18 \%$ patients (stage II) required local hemostatic measures. In none of the cases, additional measures were required.

Conclusion: There is minimum risk of bleeding in the study group which can be controlled with local measures. However, the patients should be monitored continuously. Oral antiplatelet drug need not be stopped for extraction of teeth.

Keywords: Extraction of tooth; Antiplatelet drugs; Aspirin; Bleeding

Abbreviations: OAPT: Oral Antiplatelet Therapy; CBC: Complete Blood Count; BT: Bleeding Time; CT: Clotting Time.

\section{Introduction}

Anti-platelet drugs are used for analgesics, antipyretics and as anti-platelet and anti-inflammatory drugs. Low dose antiplatelet drugs are widely used in various cardiovascular and cerebrovascular diseases. A lot of geriatric patients are on oral antiplatelet therapy (OAPT). Invasive dental therapy like Dental extraction in such patients has always been a topic of debate with respect to risk of bleeding. The literature shows sporadic case reports of life threatening bleeding. Medical physicians often advise discontinuation 
of oral antiplatelet drugs before oral surgical procedure to reduce the risk of hemorrhage. These patients are at risk of formation of thrombus resulting in life threatening risk of cardiovascular and cerebro-vascular accidents, whereas continuing of the drug has risk of hemorrhage during or after extraction. American Heart Association has recommended continuing aspirin and clopidogrel before oral surgical procedure. But still according to various surveys, majority of the practicing physicians either stop or alter antiplatelet medications before extraction. Dentists also do not prefer the risk of bleeding for their procedures.

Hence we conducted this randomized controlled trial at our institute with the following aim.

\section{Aim}

To evaluate the risk of Bleeding in Patients on continued Oral Antiplatelet Therapy undergoing extraction of Tooth.

\section{Objectives}

- To evaluate the incidence of intraoperative bleeding

- To evaluate the incidence of postoperative bleeding

- To evaluate the bleeding profile.

\section{Materials and Methods}

A Randomized controlled trial was conducted in the department of Oral and Maxillofacial Surgery of our institute. The study was approved by Institutional ethics committee.

PICO: Study population includes the patients on oral antiplatelet therapy (OAPT) who reported to our department for intra-alveolar extraction. Intervention was patients with continuing OAPT. Control group is patients with discontinuing the drug. The outcome is risk of bleeding. The sample size calculation was by EPI Info sample size calculation software by following formula: $s$

$$
\mathrm{n}=\frac{2\left(\mathrm{z}_{\alpha}+\mathrm{z}_{\beta}\right)^{2} \mathrm{p}(1-\mathrm{p})}{\mathrm{d} 2}
$$

Pilot study was conducted, where participants were divided into two groups: Control group (Group A)- 8.3\% and Study group (group B) - 31.8\%. Considering this difference, our sample size came to be 50 participants in each group. Power of study is $80 \%$ and $\alpha$ error is $95 \%$. Data collected by evaluating the patients reported to OPD of our institute. Chi square test was used for comparison and significance was kept at $\mathrm{P}$ value $<0.05$. Randomization was done by Lottery method. Statistical analysis was done by SPSS vs. 16 (Tables 1-6).

\begin{tabular}{|c|c|c|c|c|c|}
\hline & Group & N & Mean & Std. Deviation & \multirow{2}{*}{ P Value } \\
\hline \multirow{2}{*}{ AGE } & Group A & 50 & 63.4200 & 8.17685 & \multirow{2}{*}{0.11} \\
\cline { 2 - 5 } & Group B & 50 & 60.6800 & 8.86276 & \multirow{2}{*}{0.87} \\
\cline { 2 - 5 } BT & Group A & 50 & 87.8000 & 51.4599 & 0.90 .8111 \\
\hline \multirow{2}{*}{ CT } & Group B & 50 & 89.2600 & 96.4841 & 0.97 \\
\hline
\end{tabular}

Table 1: Comparison of Age, Bleeding \& Clotting time between the experimental \& control groups.

\begin{tabular}{|c|c|c|c|c|}
\hline & & \multicolumn{2}{|c|}{ Group } & \multirow{2}{*}{$P$ Value } \\
\hline & & Group A & Group B & \\
\hline \multirow{2}{*}{ Sex } & Male & 37 (74.0\%) & 37 (74.0\%) & \multirow{2}{*}{1} \\
\hline & Female & $13(26.0 \%)$ & $13(26.0 \%)$ & \\
\hline \multicolumn{2}{|c|}{ Total } & $50(100.0 \%)$ & $50(100.0 \%)$ & \\
\hline
\end{tabular}

Table 2: Sex distribution of participants between the experimental \& control groups.

\begin{tabular}{|c|c|c|c|c|}
\hline & & \multicolumn{2}{|c|}{ Group } & \multirow{2}{*}{ P Value } \\
\hline \multirow{3}{*}{ Stage I } & & Group A & Group B & \multirow{2}{*}{0.001} \\
\cline { 2 - 5 } & 0 & $19(38.0 \%)$ & $4(8.0 \%)$ & \\
\cline { 2 - 5 } & 1 & $31(62.0 \%)$ & $44(88.0 \%)$ & \\
\hline \multicolumn{2}{|c|}{ Total } & $0(.0 \%)$ & $50(100.0 \%)$ & \\
\hline
\end{tabular}

Table 3: Comparison of Bleeding after extraction at stage I between the experimental \& control groups. 


\begin{tabular}{|c|c|c|c|c|}
\hline & & \multicolumn{2}{|c|}{ Group } & \multirow{2}{*}{ P Value } \\
\hline & & Group A & Group B & \\
\hline \multirow{3}{*}{ Stage II } & 0 & $25(50.0 \%)$ & $26(52.0 \%)$ & \multirow{3}{*}{0.04} \\
\hline & 1 & $23(46.0 \%)$ & $15(30.0 \%)$ & \\
\hline & 2 & $2(4.0 \%)$ & $9(18.0 \%)$ & \\
\hline \multicolumn{2}{|c|}{ Total } & $50(100.0 \%)$ & $50(100.0 \%)$ & \\
\hline
\end{tabular}

Table 4: Comparison of Bleeding after extraction at stage II between the experimental \& control groups.

\begin{tabular}{|l|c|c|c|c|}
\hline & & \multicolumn{2}{|c|}{ Group } & \multirow{2}{*}{ P Value } \\
\hline \multirow{3}{*}{ Stage III } & & Group A & Group B & 1 \\
\cline { 2 - 5 } & 0 & $50(100.0 \%)$ & $50(100.0 \%)$ & \\
\cline { 2 - 5 } & 1 & 0 & 0 & \\
\cline { 2 - 5 } & 2 & 0 & $50(100.0 \%)$ & \\
\hline \multicolumn{2}{|c|}{ Total } & $50(100.0 \%)$ & 50 & \\
\hline
\end{tabular}

Table 5: Comparison of Bleeding after extraction at stage III between the experimental \& control groups.

\begin{tabular}{|c|c|c|c|}
\hline S.no. & Systemic diseases & Group A (n=50) & Group B (n=50) \\
\hline 1 & Myocardial Infarction & 21 & 20 \\
\hline 2 & Hypertension & 4 & 8 \\
\hline 3 & Angina Pectoris & 20 & 16 \\
\hline 4 & Cerebral stoke & 4 & 6 \\
\hline
\end{tabular}

Table 6: Data of systemic cardiac condition of the participants.

Study includes 100 number of patients with age range of 35 to 80 years. The patients were randomly divided into two groups: Group A -patients in whom oral antiplatelet drug (Study group) is continued and Group B -Patients in whom anti-platelet is stopped (Control group). Inclusion criteria includes patients between age 35 to 80 patients who are on oral antiplatelet therapy and are indicated for extraction of tooth and patients with physician fitness consent. Exclusion criteria includes patients who are unfit for extraction, patients with poor general condition and patients unwilling for consent of study.

Consent from physician for fitness for extraction was obtained for their cardiovascular condition. Informed consent was obtained from all the patients after explaining the study. Case history of all the patients was recorded. Patients' coagulation profiles were recorded including Complete blood count (CBC), Bleeding time (BT) and Clotting time (CT). Intra-alveolar extraction was performed as per indication of involved tooth under local anesthesia. Extraction socket was compressed with pressure pack and patients were observed and monitored for 24 hours. Thus, patients were observed at three stages:

Stage I: Immediately after extraction.

Stage II: 1 hour after extraction.

Stage III: 24 hour after extraction.

\section{Bleeding was graded as:}

Grade 0: No bleeding.

Grade 1: Mild bleeding which is controlled by pressure pack. Grade 2: Moderate bleeding which require local hemostatic measures.

Grade 3: Severe bleeding which is not controlled

\section{Observations}

The observations were done as per the above mentioned grading. Data was collected by clinically evaluating the patients who reported to the OPD of our institute. The mean age of the patients in the control group was 63 years whereas the mean age of the patients in the study group was 60 years .The mean Bleeding time (BT) was $1 \mathrm{~min} 30 \mathrm{sec}$ in both the groups. The mean Clotting time (CT) was 6 min 40 sec. $74 \%$ of the patients were male in both the groups 
and $26 \%$ of the patients were female. This difference is not statistically significant with respect to age, sex, BT and CT. Table 3 shows the comparison of bleeding immediately after extraction (Stage I) between control group(group A) and study group(group B) . 38\% of the patients in the control group and $8 \%$ of patients in the study group did not suffered from any bleeding. $62 \%$ of the patients in the control group and $88 \%$ patients in the study group suffered from mild bleeding which was controlled by pressure pack. Only $4 \%$ patients in the study group suffered from moderate bleeding which required local haemostatic measures. Bleeding was controlled by gauze impregnated with feracrylum solution. This difference is statistically significant between both the groups $(\mathrm{P}=0.001)$. Table 4 shows incidence of bleeding 1 hour after extraction (Stage II). 50\% patients in control group and $52 \%$ of patients in study group showed controlled bleeding 1 hour after extraction. $46 \%$ patients in control group and 30\% patients in study group suffered mild bleeding. Bleeding could be completely controlled with pressure pack application alone. Only $4 \%$ of the patients in the control group and $18 \%$ of the patients in the study group had moderate bleeding which required local haemostatic measures like feracrylum pack. Bleeding differed significantly between two groups ( $\mathrm{P}$ $=0.04$ ). Table 5 shows comparison of bleeding 24 hours after extraction (Stage III). None of the patients in both the groups suffered from any bleeding 24 hours after extraction of tooth which was statistically insignificant $(\mathrm{P}=1.00)$.

\section{Discussion}

Antiplatelet drugs are also widely used as antiinflammatory, analgesics, and antipyretics. Antiplatelet effect is achieved at low doses whereas anti-inflammatory effect requires higher doses. Antiplatelet drugs irreversibly inhibit the cyclooxygenase pathway and thereby inhibit the release of thromboxane. Thus, it affects platelet aggregation and platelet plug formation. Therefore, bleeding time is prolonged. Antiplatelet therapy is widely used in various cardiovascular and cerebrovascular accidents. Oral surgical procedures in such patients are always controversial as many of the practicing physicians recommend discontinuation of aspirin and clopidogrel before dental extraction contrary to the recommendation of the American Heart Association (Figure 1).

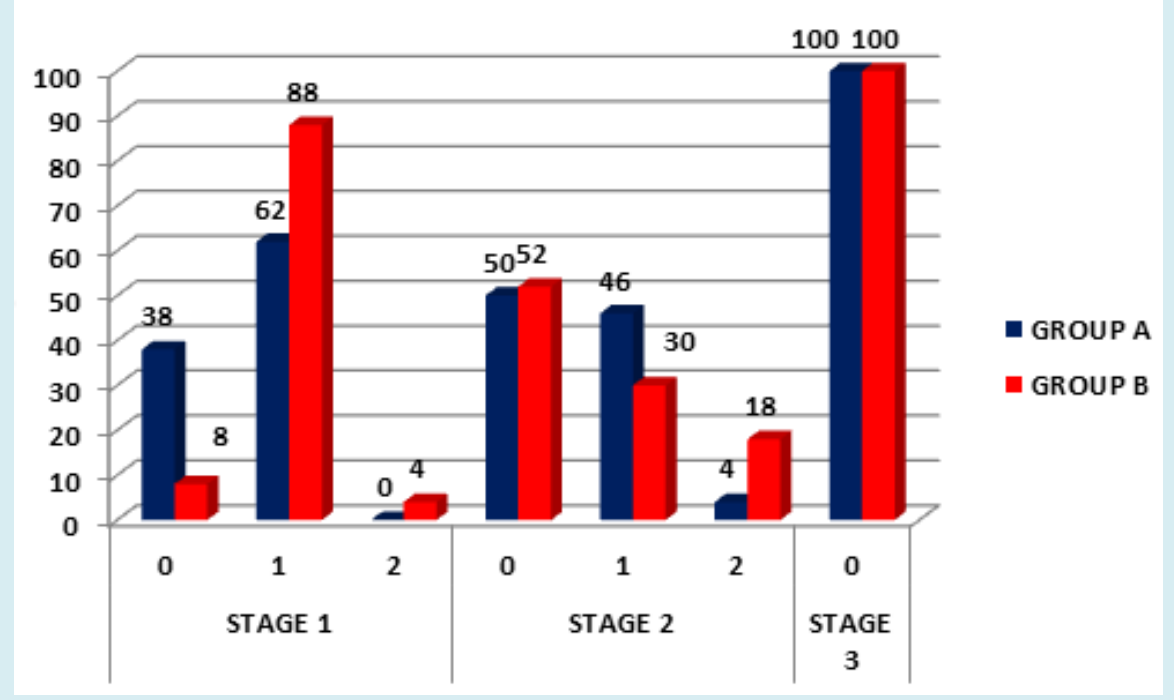

Figure 1: Comparison of Bleeding after extraction between the experimental \& control groups.

Murphy J, et al. [1] conducted a survey to assess the approach of dental practitioners in the management of patients taking warfarin and antiplatelet drugs. The results showed that $86 \%$ of dental practitioners advised their patients to stop oral antiplatelet drugs before dental extraction. Krishnan B, et al. [2] conducted a prospective clinical study with a sample size of 82 patients requiring dental extraction. Patients continuing/discontinuing aspirin were compared with healthy individuals. The results of the study conclude that routine dental extraction can be safely performed in patients on long term antiplatelet therapy without any interruption or alteration of their medication. Madan GA, et al. [3] conducted a clinical trial in 51 patients who were on long term low dose aspirin therapy and concluded that minor oral surgical procedures can be safely carried out without stopping long term low dose aspirin therapy. These results are consistent with our study. Mohsen Sadeghi, et al. [4] compared 64 patients on aspirin and Plavix (Clopidogrel) with 50 healthy patients and concluded that using aspirin and plavix simultaneously have no considerable effect on 
the risk of bleeding in patients undergoing conventional forceps extraction of a single tooth. Bajkin, et al. [5] conducted a prospective clinical study with 160 patients to evaluate the effects of single and dual antiplatelet treatment on postoperative bleeding having dental extractions and compared with healthy individuals. They concluded that patients taking single or dual antiplatelet drugs can safely undergo dental extraction without interruption of treatment using local haemostatic measures.

Lillis, et al. [6] conducted a prospective study to assess the risk of immediate and late onset bleeding complications during uninterrupted single or dual antiplatelet therapy in patients undergoing dental extractions. Study participants ( $n=643$ ) were categorized as treatment group ( $n=111)$ receiving uninterrupted aspirin and /or clopidogrel and a control group $(n=532)$ receiving no antiplatelets. They concluded that dental extractions can be safely performed in patients receiving single or dual OPT when appropriate local hemostatic measured are taken. In contrast to these studies, ours was a randomized control trial in which patients were equally and randomly divided into two groups. In conclusion, dental extractions can be safely performed in individuals taking oral antiplatelet drugs without any alteration in their medication regime.

\section{Limitations}

The main limitation of this study is it did not evaluate the episode of thrombo-embolism in the control group. Single and double antiplatelet drugs patients were not divided into two groups.

\section{Conclusion}

We conclude that there is minimum risk of bleeding in patients in whom oral antiplatelet therapy is continued while undergoing intra-alveolar extraction. However, the patients should be monitored continuously. The incidence of bleeding should be identified and controlled with local haemostatic measures like feracrylum, gelfoam and suture material. Oral antiplatelet drug need not be stopped before routine extraction of tooth.

\section{References}

1. Murphy J, Twohig E, Mcwilliams SR (2010) Dentists approach to patients on anti-platelet agents and warfarin: a survey of practice. J Ir Dent Assoc 56(1): 2831.

2. Krishnan B, Shenoy NA, Alexander M (2008) Exodontia and antiplatelet therapy. J Oral Maxillofac Surg 66(10): 2063-2066.

3. Madan GA, Madan SG, Madan G, Madan AD (2005) Minor Oral Surgery without stopping daily low dose aspirin therapy: A study of 51 patients. J Oral Maxillofac Surg 63(9): 1262-1265.

4. Ghahrody MS, Malekshah SHY, Sari HK, Yazdanpanah $\mathrm{H}$, Zavareh MSR, et al. (2016) Bleeding after tooth extraction in patients taking aspirin and clopidogrel (Plavix) compared with healthy controls. Br J Oral Maxillofac Surg 54(5): 568-572.

5. Bajkin BV, Urosevic IM, Stankov KM, Petrovic BB, Bajkin IA (2015) Dental extractions and risk of bleeding in patients taking single and dual antiplatelet treatment. Br J Oral Maxillofac Surg 53(1): 39-43.

6. Lillis T, Ziakas A, Koskinas K, Tsirlis A, Giannoglou G (2011) Safety of Dental Extractions during Uninterrupted Single or dual Antiplatelet Treatment. Am J Cardiol 108(7): 964-967. 